\title{
Definition and Assessment of Reference Values for PMU Calibration in Static and Transient Conditions
}

\author{
Guglielmo Frigo, Claudio Narduzzi \\ Department of Information Engineering \\ University of Padova, Padova, Italy \\ frigogug@dei.unipd.it
}

\author{
Daniele Colangelo, Marco Pignati, Mario Paolone \\ Distributed Electrical Systems Laboratory (DESL) \\ École Polytechnique Fédérale de Lausanne (EPFL), Switzerland \\ daniele.colangelo@epfl.ch
}

\begin{abstract}
The calibration of Phasor Measurement Units (PMUs) consists of comparing Coordinated Universal Time (UTC) timestamped phasors (synchrophasors) estimated by the PMU under test, against reference synchrophasors generated through a PMU calibrator. The IEEE Standard C37.118-2011 and its amendment (IEEE Std) describe compliance tests for static and dynamic conditions, and indicate the relative limits in terms of accuracy. In this context, the paper focuses on the definition and accuracy assessment of the reference synchrophasors in the test conditions dictated by the above IEEE Std. In the first part of the paper, we describe the characterization of a nonlinear least-squares (NL-LSQ) fitting algorithm used to determine the parameters of the reference synchrophasors. We analyse the uniqueness and robustness of the solution provided by the algorithm. We assess its accuracy within the whole range of static tests required by the IEEE Std. In the second part, we discuss the appropriateness of synchrophasor model to evaluate the PMU performance in step test conditions. We compare the proposed algorithm against two synchrophasor estimation algorithms. Finally, we propose a time domain process for the better evaluation of PMU performances in transient conditions.
\end{abstract}

\section{INTRODUCTION}

The IEEE Standard for Synchrophasor Measurements for Power Systems C37.118.1-2011 [1] and its amendment [2] (henceforth called IEEE Std) indicate compliance limits for a Phasor Measurement Unit (PMU) operating in static and dynamic conditions. The accuracy requirements for PMUs are expressed through Total Vector Error (TVE), Frequency Error (FE), and Rate-Of-Change-Of-Frequency Error (RFE). As for any other measurement device, the compliance certification and the calibration of a PMU, with respect to the limits imposed by the related IEEE Std, is performed by comparing the PMU under test with a reference system (PMU calibrator) that, in general, has a level of accuracy at least one order of magnitude better. In particular, Coordinated Universal Time (UTC) tagged synchrophasors estimated by the PMU under test are compared against reference synchrophasors whose uncertainty (in terms of TVE, FE and RFE) is known with a much better accuracy than the device under test.

The scalability of PMUs to active distribution networks (ADNs) requires a constantly increasing level of accuracy of such devices. As a consequence, the metrological characterization of PMU calibrators and the algorithms used to generate the reference synchrophasors associated to the tests of the IEEE Std, is an open issue for National Metrology Institutes (NMIs) and research groups [3]-[6]. Indeed, the recent literature is proposing synchrophasor estimation algorithms characterized by a theoretical TVE in the order of $0.0 x \%$. For this reason, in the first part of the article, we propose a thorough characterization of a non-linear least-squares (NL-LSQ) fitting algorithm used to define the reference synchrophasors for the static compliance tests of the IEEE Std. The aim is to guarantee a TVE significantly smaller than the synchrophasor estimation algorithm performances.

We study the optimization problem solved by the NLLSQ algorithm in terms of solution uniqueness and robustness against noise, and we fully characterize the algorithm for the static tests imposed by the IEEE Std. At this stage of the work, we perform the analysis using synthetic waveforms.

The second part of the article discusses on the validity of synchrophasor definition when a rapid variation of amplitude, frequency or phase occurs. Indeed, the definition of synchrophasor does not hold in presence of particular transient conditions characterized by a wide-band spectrum. We discuss on the limits of the synchrophasor definition making specific reference to a amplitude step test where we change, intentionally, the dynamic of the event. We compare the response of the NL-LSQ algorithm against two representative PMU algorithms based on Discrete Fourier Transform (DFT) [7], [8], and we propose a time-domain comparison between the PMUestimated phasor and a non-stationary known signal model.

The paper is structured as follows: in Section II, after the problem statement, we study the uniqueness, stability and robustness of the solution of the NL-LSQ algorithm; we report in Section III the performance assessment of the algorithm in the static test conditions provided by the IEEE Std; in Section IV, we discuss on the validity of the synchrophasor definition in specific transient conditions and we illustrate an alternative method for the performance assessment of PMUs directly in time domain. In the final Section, we provide some concluding remarks and we illustrate the future steps of this research.

\section{Problem Statement}

We can describe a generic time-variant noise-less power signal affected by disturbances through the following model:

$$
x(t)=A\left(1+\varepsilon_{A}(t)\right) \cdot \cos \left(2 \pi f t+\varphi_{0}+\varepsilon_{\varphi}(t)\right)+\eta(t)
$$

where $A, f$ and $\varphi_{0}$ are the amplitude, the fundamental frequency and the initial phase of the signal, respectively. The time-varying terms $\varepsilon_{A}(t)$ and $\varepsilon_{\varphi}(t)$ represent amplitude and phase fluctuations. The term $\eta(t)$ models possible sources of spurious contributions, accounting in particular for harmonic or inter-harmonic components, DC components and, in general, 
any transient conditions. Focusing on the context of PMU calibration, the ideal phasor associated to the fundamental component is uniquely identified in (1) by the triplet:

$$
\mathcal{P}^{i d}=\left\{A^{i d}, f^{i d}, \varphi_{0}^{i d}\right\}
$$

that is the set of constant ideal parameter values for the amplitude $A^{i d}$, frequency $f^{i d}$ and initial phase $\varphi_{0}^{i d}$. We assume that the time-varying fluctuations $\varepsilon_{a}(t)$ and $\varepsilon_{\varphi}(t)$, as well as the disturbances due to harmonic or inter-harmonic components $\eta(t)$ are known a priori, so that the nominal signal $x(t)$ is fully defined. In practice, $x(t)$ is known through a discrete time-series of measurements, defined as follows:

$$
x[n]:=\left\{x(t)+\rho(t) \quad \mid \quad t=n T_{s}, n=\left[1, \ldots N_{m}\right] \in \mathbb{N}\right\}
$$

where $N_{m}$ is the number of samples that compose $x[n]$, and the additional term $\rho(t)$ accounts for wide-band disturbances, e.g additive uncorrelated noise. For the sake of simplicity, we assume a uniform sampling step $T_{s}$. Given $x[n]$, we can estimate the actual signal parameter triplet $\hat{\mathcal{P}}=\left\{\hat{A}, \hat{f}, \hat{\varphi}_{0}\right\}$ by solving the following non-linear least-squares problem:

$$
\operatorname{argmin}_{\hat{\mathcal{P}}}\|x[n]-\hat{x}[n]\|_{2} \quad \text { s.t. } \quad \hat{\mathcal{P}} \in \mathcal{S}
$$

where $\hat{x}[n]$ is the time-series of the signal we can reconstruct from the solution $\hat{\mathcal{P}}$, and $\mathcal{S}$ defines a specific region of feasible values for $A, f$ and $\varphi_{0}$ (see Section III). The estimated values of the problem (4) are used to generate the reference synchrophasors that are compared with those provided by the PMU under test when it is fed with the signal $x(t)$.

The problem (4) is strictly non-convex, so it cannot provide a solution in closed form. For this purpose, we solve the problem through an iterative optimization procedure. Starting from an initial guess $\mathcal{P}^{*}=\left\{A^{*}, f^{*}, \varphi_{0}^{*}\right\}^{1}$, the estimated values of $\hat{\mathcal{P}}$ are iteratively updated solving the problem (4) until the energy of the residual is comparable with the expected noise level. In particular, the non-linear least-squares (NLLSQ) fitting algorithm we use for our calculations, is based on the the well-known Levenberg-Marquardt algorithm [9].

The signal model in (1) enables us to reproduce any test condition of the IEEE Std, as well as other realistic operating conditions, like transient phenomena. The proposed NL-LSQ algorithm introduces a single constraint: as the cardinality of $\hat{\mathcal{P}}$ is equal to three, $x[n]$ must consist of at least three samples.

\section{CHARACTERIZATION OF NL-LSQ ALGORITHM}

As mentioned above, the objective function defined in (4), is strictly non-linear and non-convex. Depending on $\mathcal{P}^{*}$, during the optimization procedure the iteratively updated solution $\hat{\mathcal{P}}$ can end up in a local minimum which does not coincide with the absolute minimum $\hat{\mathcal{P}}^{\text {min }}$. In other words, varying the initial guess $\mathcal{P}^{*}$, the optimization procedure may present multiple solutions. For this reason, we study in this Section the uniqueness, the accuracy and the robustness of the solution, as function of the observation interval length and with respect to additive wide-band noise injection.

\section{A. Solution Uniqueness}

A thorough study of the objective function trend enables us to define a confidence interval for the initial guess $\mathcal{P}^{*}$ which

\footnotetext{
${ }^{1}$ In the context of PMU calibration, it is reasonable to assume that we can infer $\mathcal{P}^{*}$ directly from the nominal parameter values of $x(t)$.
}

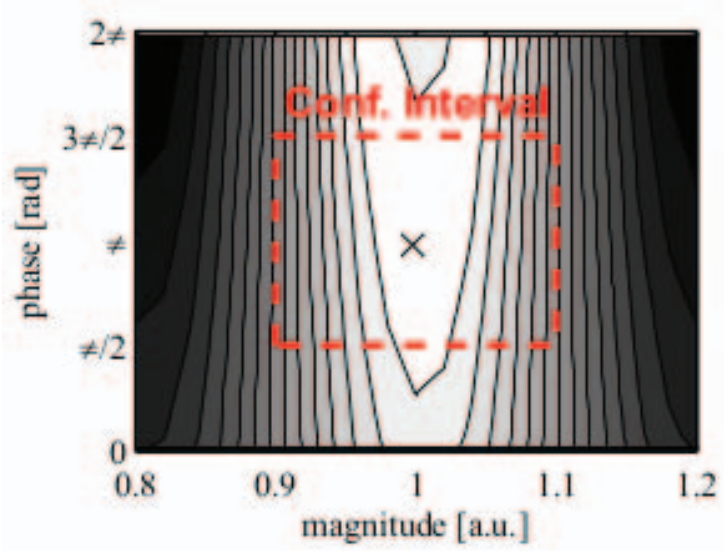

Fig. 1. Objective function trend. In the considered range, the function is convex, and the global minimum $\hat{\mathcal{P}}^{\text {min }}$ (cross) is the best possible approximation of $\mathcal{P}^{i d}$. The confidence interval (red dotted line) guarantees the convergence to the unique solution.

ensures uniqueness of the solution. For sake of simplicity, let's consider a time-series $x[n]$ in nominal steady-state conditions:

$$
x[n]=\sqrt{2} A^{r m s} \cdot \cos \left(2 \pi f n T_{s}+\varphi_{0}\right)
$$

whose equivalent synchrophasor formulation is given by:

$$
\begin{aligned}
X[n] & =A^{r m s} \cdot \mathrm{e}^{j \Phi} \\
\Phi & =2 \pi f n T_{s}+\varphi_{0}
\end{aligned}
$$

where $A^{r m s}$ is the root mean square magnitude of the fundamental component, and the synchrophasor phase $\Phi$ takes into account both frequency and initial phase contribution.

In the present case, we assume the amplitude is equal to 1 a.u., the instantaneous frequency is $50 \mathrm{~Hz}$, and we set the initial phase to $0 \mathrm{rad}$. We adopt a sampling frequency of $5 \mathrm{kHz}$, and the observation interval length consists of three cycles at the nominal frequency, i.e. $60 \mathrm{~ms}$ (corresponding to 300 samples). Following usual practice, we refer the solution $\hat{\mathcal{P}}$ to the observation interval midpoint, i.e. $n T_{s}=30 \mathrm{~ms}$. As a consequence, the synchrophasor phase $\Phi$ is equal to $\pi$. We solve numerically the problem (4) while varying $\mathcal{P}^{*}$ in a plausible range of the parameters domain. In particular, we vary $A^{*}$ from 0.8 and 1.2 a.u., $f^{*}$ from 45 to $55 \mathrm{~Hz}$, and $\varphi_{0}^{*}$ around $\pm \pi / 2 \mathrm{rad}$. In the context of calibration, we can assume that the generated parameter value are very close to the their initial guess. As a consequence, it is reasonable to use as initial guess the values set by the user of the calibrator.

In general, due to inherent non-convexity issues a unique solution cannot be found. However, if we restrict the range of the feasible values $\mathcal{S}$, the objective function exhibits a convex feature and $\hat{\mathcal{P}}^{\text {min }}$ (black cross in Fig. 1) is the best possible estimation of $\mathcal{P}^{\text {nom }}$. We perform the analysis using a nominal steady-state signal, but similar conclusions hold for the other test conditions of the IEEE Std. Even with a more complex signal model, in a restricted neighbourhood of the parameter nominal values, the objective function is nearly convex.

We study the influence of $\mathcal{P}^{*}$ on the solution of the NLLSQ algorithm through a Monte Carlo analysis. In particular, we evaluate the combined effect of the three initial guess values $A^{*}, f^{*}$ and $\varphi_{0}^{*}$ on the solution convergence to the ideal values in $\mathcal{P}^{i d}$. For each parameter defined in $\mathcal{P}^{*}$, we divide the range 
TABLE I. Fitting ACCURACY VS OBSERVATION INTERVAL LENGTH

\begin{tabular}{|c|c|c|c|c|}
\hline Samples & Parameter & Mean Dev $\mu$ & Std Dev $\sigma$ & TVE [\%] \\
\hline \multirow{3}{*}{$300(60 \mathrm{~ms})$} & $A$ [a.u.] & $-3,58$ E-9 & $2.39 \mathrm{E}-8$ & \multirow{3}{*}{9.27 E-6 } \\
\hline & $f[\mathrm{~Hz}]$ & 5.97 E-10 & 3.04 E-8 & \\
\hline & $\varphi_{0}[\mathrm{rad}]$ & -1.86 E-9 & 2.53 E-8 & \\
\hline \multirow{3}{*}{$500(100 \mathrm{~ms})$} & $A$ [a.u.] & $-3,61$ E-9 & $1.69 \mathrm{E}-8$ & \multirow{3}{*}{7.48 E-6 } \\
\hline & $f[\mathrm{~Hz}]$ & $2.56 \mathrm{E}-10$ & $1.05 \mathrm{E}-8$ & \\
\hline & $\varphi_{0}[\mathrm{rad}]$ & -1.77 E-9 & 2.03 E-8 & \\
\hline \multirow{3}{*}{$1000(200 \mathrm{~ms})$} & $A$ [a.u.] & $-4,02$ E-9 & 1.07 E-8 & \multirow{3}{*}{6.37 E-6 } \\
\hline & $f[\mathrm{~Hz}]$ & $4.06 \mathrm{E}-11$ & 4.49 E-9 & \\
\hline & $\varphi_{0}[\mathrm{rad}]$ & -1.56 E-9 & $1.92 \mathrm{E}-8$ & \\
\hline
\end{tabular}

in 21 uniformly spaced values. Therefore, we perform the Monte Carlo analysis on a total of 9261 possible combinations of initial guess values. For each possible combination, we solve the optimization problem and, as a criterion for success probability, we consider a trial successful if the solution $\hat{\mathcal{P}}$ deviates from the ideal $\mathcal{P}^{i d}$ of a TVE lower than $0.001 \%$. According to this success criterion, the confidence interval is limited between 0.9 to 1.1 a.u. in terms of $A^{r m s}$ and between $\pi / 2$ and $3 / 2 \pi$ rad in terms of $\Phi$. It is worth noticing that $\Phi$ between $\pi / 2$ and $3 / 2 \pi$ rad corresponds to limit $f$ between 48 to $52 \mathrm{~Hz}$ and $\varphi_{0}$ between $\pm \pi / 4$. In this confidence interval, the NL-LSQ fitting algorithm converges to the global minimum $\mathcal{P}^{\text {min }}$ also in presence of an inaccurate initial guess $\mathcal{P}^{*}$.

\section{B. Solution Accuracy and Robustness}

This subsection focuses on the solution robustness with respect to additive uncorrelated noise injection, and the solution accuracy as function of the observation interval length.

In nominal steady-state conditions, the solution $\hat{\mathcal{P}}$ coincides with the nominal reference values $\mathcal{P}^{i d}$ except for the numerical uncertainty introduced by the computing system. The scenario changes when additive noise is considered. Real measurements are affected by noise, e.g. due to the non-idealities inherent in the acquisition system, and a wide-band noise limits, in general, the accuracy of the solution. It is worth noticing, although the noise is unknown a priori (see Section II), in the context of PMU calibration the uncertainty deriving from its contribution can be fully characterized. To a first approximation, additive noise can represent also the effect of other non-idealities, such as time synchronization jitter or phase noise. A more detailed analysis will be considered in future steps of the research.

In order to evaluate the algorithm robustness against noise, we analyse a nominal steady-state power signal to which white Gaussian noise at different energy levels has been intentionally added. For this analysis, we generate $6 \mathrm{~s}$ of signal and we consider 200 observation intervals or, equivalently, 200 synchrophasors. In order to reproduce a reporting rate of 50 fps, hence, the synchrophasors are partially overlapped by 40 ms. Given a sampling frequency of $5 \mathrm{kHz}$, the signal-to-noise ratio (SNR) ranges from 60 to $140 \mathrm{~dB}$.

The estimation uncertainty of $\hat{\mathcal{P}}$ can be expressed in terms of mean deviation $\mu$ and standard deviation $\sigma$. Using

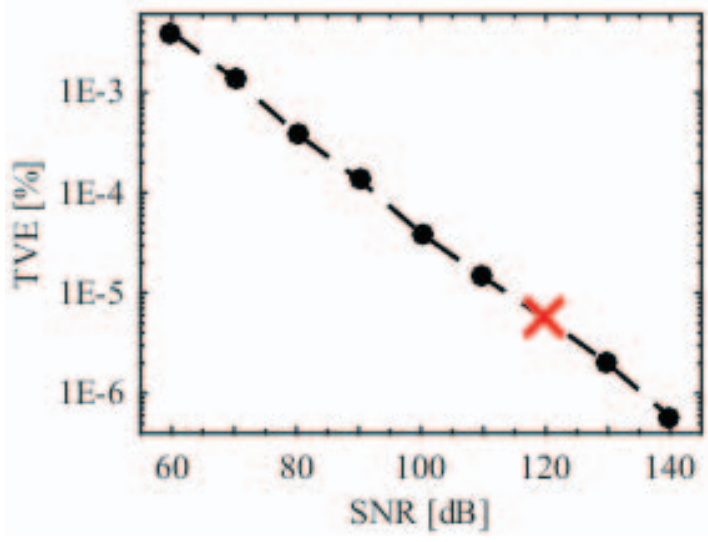

Fig. 2. Solution TVE function of the SNR associated to the additive noise The TVE decreases when SNR increases and it follows an almost-linear trend The red cross indicates a plausible SNR value in a context of PMU calibration.

the synchrophasor definition of (2), we associate the TVE to the performance of the NL-LSQ algorithm by considering a conservative range of $\pm 3 \sigma$ for each signal parameter. Figure 2 shows that TVE decreases when the SNR increases and it follows an almost-linear trend. In our current set-up, we employ ADCs with 18-bits resolution [5] and we experimentally characterize a SNR around $115 \mathrm{~dB}$ (full scale $\pm 10 \mathrm{~V}$ ).

To study the influence of the sample number $N_{m}$ on the accuracy of the solution, we vary the observation intervals on which the 200 synchrophasors are defined. In Table I, we report the performance of the fitting algorithm for different observation interval lengths, namely $60 \mathrm{~ms}, 100 \mathrm{~ms}$ and $200 \mathrm{~ms}$ corresponding to 300,500 and 1000 samples, respectively. As expected, TVE decreases as the number of samples increases. In the following sections, we refer to an observation interval length of 300 samples, i.e. $60 \mathrm{~ms}$, even though it must be remembered that the proposed fitting algorithm can test PMUs at different observation interval lengths.

\section{IEEE C37.118.1 StATIC TESTS}

In this section, we describe the characterization of the NL-LSQ algorithm in the static test conditions provided by IEEE Std, namely nominal (NOM) and off-nominal static (ONS), harmonic distortions (HD), and out-of-band (OOB) or inter-harmonic distortions. In the NOM test, we employ for amplitude, frequency, and initial phase the ideal values, namely $\mathcal{P}^{i d}=\{1,50,0\}$. In the ONS tests, we vary $f^{i d}$ from 45 to $55 \mathrm{~Hz}, A^{i d}$ from 0.8 to 1.2 a.u., and $\varphi_{0}^{i d}$ from $-\pi$ to $\pi$ rad.

In the HD and OOB tests, we define the distortion level through the total harmonic distortion (THD) index:

$$
T H D=\sum_{h} \frac{\sqrt{A_{h}^{2}}}{A^{r m s}}
$$

where $A_{h}$ is the RMS magnitude of $h$-th harmonic or interharmonic component. We consider a single interferring component corresponding to a THD equal to $10 \%$, i.e. the maximum distortion level required by the IEEE Std, and we vary its frequency. In this way, we can evaluate the influence of the frequency deviation between fundamental and interferring component on the algorithm performances. As in Section III, 
TABLE II. NL-LSQ Estimation ACCURACY IN STATIC TESTS

\begin{tabular}{|c|c|c|c|c|}
\hline Test & Parameter & Mean Dev $\mu$ & Std Dev $\sigma$ & TVE $[\%]$ \\
\hline \multirow{3}{*}{ NOM } & $A$ [a.u.] & $-3,58$ E-9 & 2.39 E-8 & \multirow{3}{*}{9.27 E-6 } \\
\hline & $f[\mathrm{~Hz}]$ & 5.97 E-10 & 3.04 E-8 & \\
\hline & $\varphi_{0}[\mathrm{rad}]$ & $-1.86 \mathrm{E}-9$ & $2.53 \mathrm{E}-8$ & \\
\hline \multirow{3}{*}{$\begin{array}{c}\text { ONS } \\
(-\pi \mathrm{rad})\end{array}$} & $A$ [a.u.] & 3,19 E-9 & $2.56 \mathrm{E}-8$ & \multirow{3}{*}{1.19 E-5 } \\
\hline & $f[\mathrm{~Hz}]$ & -3.05 E-9 & $3.03 \mathrm{E}-8$ & \\
\hline & $\varphi_{0}[\mathrm{rad}]$ & $-1.88 \mathrm{E}-9$ & $3.62 \mathrm{E}-8$ & \\
\hline \multirow{3}{*}{$\begin{array}{c}\text { HD } \\
(100 \mathrm{~Hz})\end{array}$} & $A$ [a.u.] & 4.48 E-9 & 2.66 E-8 & \multirow{3}{*}{2.48 E-5 } \\
\hline & $f[\mathrm{~Hz}]$ & -1.43 E-9 & $3.09 \mathrm{E}-8$ & \\
\hline & $\varphi_{0}[\mathrm{rad}]$ & -1.86 E-9 & 8.44 E-8 & \\
\hline \multirow{3}{*}{$\begin{array}{c}\text { OOB } \\
(25 \mathrm{~Hz})\end{array}$} & $A$ [a.u.] & 6.63 E-9 & $2.52 \mathrm{E}-8$ & \multirow{3}{*}{2.39 E-5 } \\
\hline & $f[\mathrm{~Hz}]$ & -1.47 E-9 & $2.80 \mathrm{E}-8$ & \\
\hline & $\varphi_{0}[\mathrm{rad}]$ & -4.13 E-9 & 8.11 E-8 & \\
\hline \multirow{3}{*}{$\begin{array}{c}\text { OOB } \\
(75 \mathrm{~Hz})\end{array}$} & $A$ [a.u.] & 3.01 E-9 & $2.47 \mathrm{E}-8$ & \multirow{3}{*}{2.41 E-5 } \\
\hline & $f[\mathrm{~Hz}]$ & $1.59 \mathrm{E}-10$ & $1.92 \mathrm{E}-8$ & \\
\hline & $\varphi_{0}[\mathrm{rad}]$ & 7.99 E-9 & $2.35 \mathrm{E}-8$ & \\
\hline
\end{tabular}

we assume a sampling frequency of $5 \mathrm{kHz}$, an observation interval of $60 \mathrm{~ms}$, and a SNR equal to $120 \mathrm{~dB}$.

In Table II, we report the results for all static tests. For the sake of brevity, the table shows only the worst ONS case corresponding to an initial phase of $-\pi$ rad. For each parameter, we calculate the mean and standard deviation. As it can be seen, the corresponding TVE is around $0.10 \mathrm{ppm}$ for all considered test conditions.

In the HD test, the fitting algorithm is more accurate when higher harmonics are added to the nominal signal (Fig. 3). In the worst case, the TVE does not exceed $0.30 \mathrm{ppm}$. In the OOB test, we characterize the algorithm in the presence of inter-harmonic components from 5 to $25 \mathrm{~Hz}$, and from 75 to $95 \mathrm{~Hz}$ with steps of $5 \mathrm{~Hz}$ (Fig. 4). The fitting algorithm proves to be sufficiently resilient against OOB interferences. We can assume that the accuracy of the algorithm is nearly constant outside the passband 25 to $75 \mathrm{~Hz}$ (see the vertical axis scale in Fig. 4). In particular, we calculate a TVE in the order of $0.30 \mathrm{ppm}$

In all the considered conditions, the FE does not exceed $0.1 \mu \mathrm{Hz}$, that corresponds to a RFE of $10 \mu \mathrm{Hz} / \mathrm{s}$. We calculate the RFE as the maximum deviation between two consecutive frequency estimations, i.e. two times the FE, divided by $20 \mathrm{~ms}$ (corresponding to a reporting rate of $50 \mathrm{fps}$ ).

\section{ON SYNCHROPHASOR DEFINITION IN TRANSIENT CONDITIONS}

In this section, we discuss on the appropriateness of synchrophasor definition when a step-like transient occurs. The IEEE Std describes the parameters related to the fundamental component of a generic test signal, through a synchrophasor representation in frequency domain. As they account for deviations from the nominal steady-state condition, synchrophasor magnitude and phase are, in general, functions of time.

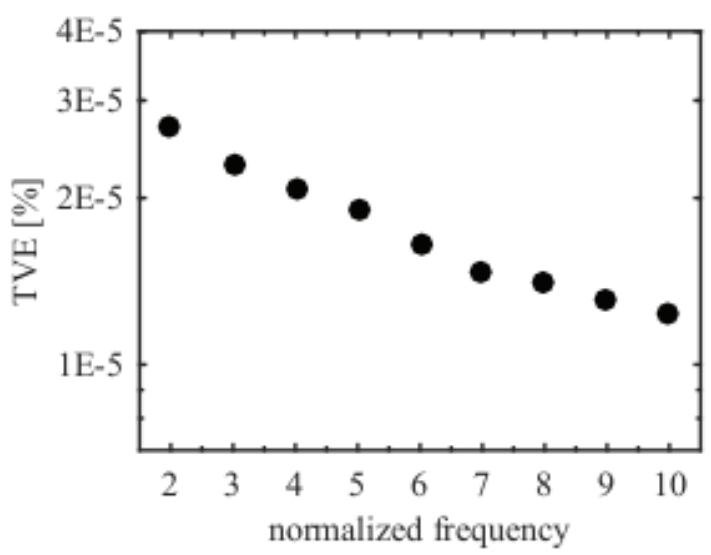

Fig. 3. TVE function of the normalized frequency (i.e. harmonic order) of the interferring component in HD test. The TVE decreases when the interferring component is far from the fundamental frequency

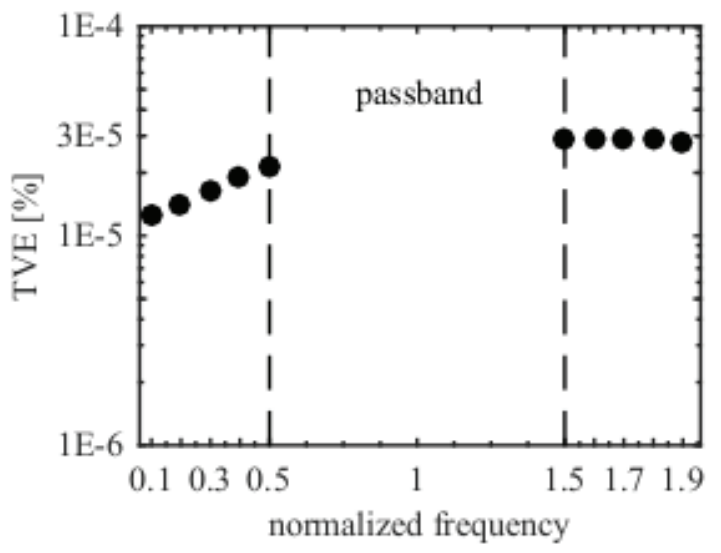

Fig. 4. TVE function of the normalized frequency of the interferring component in OOB test. Outside the passband dictated by the IEEE Std, the TVE is below $0.30 \mathrm{ppm}$.

The synchrophasor representation relies on the assumption that the test signal has a spectrum composed by narrow-band frequency components. In other words, IEEE Std assumes that the DFT representation of a power signal consists of a term associated to the fundamental component, plus a restricted set of coefficients associated to eventual harmonic or inter-harmonic contributions. Nevertheless, the finite spectrum assumption might not be able to represent rapid variations of the signal parameters with a satisfying level of accuracy. As a consequence, also the definition of TVE might become inconsistent and it would not represent the best metric for the performance assessment of a generic PMU. In this context, we discuss on the appropriateness of the synchrophasor definition by making specific reference to amplitude step conditions.

In the presence of an instantaneous variation of amplitude, the signal $x(t)$ can be modelled as follows:

$$
x(t)=A\left(1+A_{T} \cdot \frac{1}{1+\exp ^{-k\left(t-T_{T}\right)}}\right) \cdot \cos \left(2 \pi f t+\varphi_{0}\right)
$$

where $A_{T}$ and $T_{T}$ account for step depth and step occurrence time, respectively. The slope parameter $k$ determines the dynamic of the step. In the current analysis, we consider 


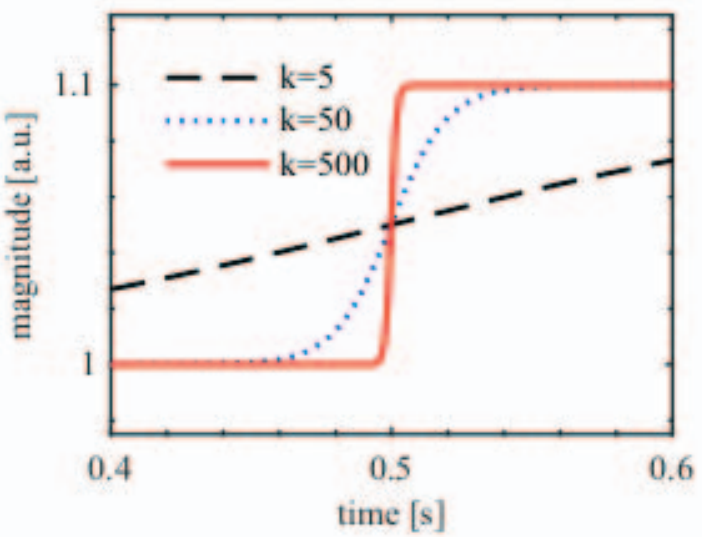

Fig. 5. RMS value of the amplitude step signal for different $k$ values. The step dynamic increases with $k$. An instantaneous amplitude variation is obtained when $k$ tends to infinity.

$A_{T}=0.1$ and $T_{T}=0.48$ s. As shown in Fig. 5, small $k$ values correspond to slow and smooth amplitude variations, whereas high $k$ values, with respect to the considered reporting rate of $50 \mathrm{fps}$, correspond to quasi-instantaneous variations. This signal model enables us to evaluate the influence of different step dynamics on the PMU performance. In other words, we can evaluate the PMU response with different test signal bandwidths.

We compare the results obtained using the NL-LSQ algorithm against two representative synchrophasor estimation algorithms. The two selected estimation algorithms are the enhanced interpolated DFT (IP-DFT) [7] and the compressive sensing-based Taylor Fourier model (CS-TFM) [8]. They provide a rather comprehensive overview of DFT-based approaches. The IP-DFT is based on a static signal model and minimizes the leakage interfering contribution, whereas the CS-TFM is based on a dynamic signal model and exploits a Taylor-Fourier expansion to model time-variable conditions.

In this analysis, the signal has a duration of $1 \mathrm{~s}$. We set the signal parameters $\left(A, f, \varphi_{0}\right)$ to their nominal values, and we vary $k$ from 5 to 1000 . The fitting algorithm adopts the Eq. (8) as signal model, where the initial guess values $A_{T}, T_{T}$ and $k$ are known a priori. As mentioned in Section III-B, we divide the signal into observation intervals of $60 \mathrm{~ms}$ with a reporting rate of $50 \mathrm{fps}$, the sampling frequency is equal to $5 \mathrm{kHz}$, and SNR is set to $120 \mathrm{~dB}$.

We report in Fig. 6 the maximum TVE, obtained by using the three algorithms, as function of $k$ values, for two different observation interval lengths, namely 300 and 500 samples (60 ms and $100 \mathrm{~ms}$ ). The TVE computed with IPDFT and CS-TFM degrades as $k$ increases. In particular, with $k \geq 50$, the TVE increases rapidly, and in the case of IP-DFT exceeds the limit of $1 \%$ dictated by the IEEE Std. While the accuracy of DFT-based algorithms depends on the dynamic of the event, the accuracy of NL-LSQ algorithm is nearly constant and is comparable to the performance provided in static test conditions. This discrepancy is due to the fact that NL-LSQ algorithm implements the exact signal model of Eq. (8), whereas IP-DFT and CS-TFM adopt the canonical synchrophasor representation. Moreover, the TVE computed with IP-DFT and CS-TFM depends on observation interval length, whereas the influence of the observation interval on the

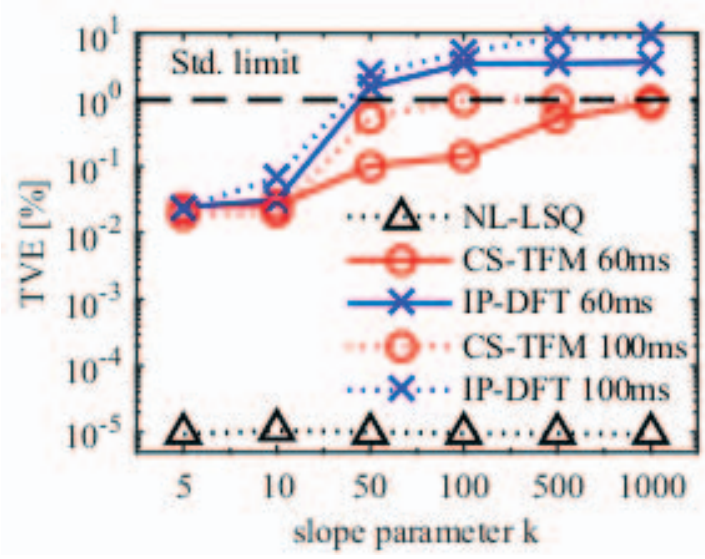

Fig. 6. Maximum TVEs function of $k$ obtained for different observation intervals (60 and $100 \mathrm{~ms}$ ). The accuracy of IP-DFT and CS-TFM degrades with $k$. NL-LSQ shows a nearly constant performance abound $0.1 \mathrm{ppm}$.

NL-LSQ algorithm is negligible. As the NL-LSQ algorithm implements the exact signal model, it is not affected by the limitations of DFT-based algorithms and can generate very accurate synchrophasor reference values for the calibration of PMUs, also in transient conditions.

However, the TVE comparison provides only a partial overview of the phenomena under investigation. In fact, according to TVE comparison, the CS-TFM algorithm is compliant to IEEE Std, but TVE still does not provide enough information on the accuracy with which the algorithm follows the transient event. As we already mentioned, during a transient event, the finite discrete spectrum model assumption does not hold anymore. In particular, as the signal bandwidth broadens it fits less and less accurately into a finite discrete spectrum model. In order to better explain this statement, Fig. 7 compares the spectrum of an amplitude step signal (STEP), for $k=1000$, in the neighbourhood of $T_{T}$, with the spectrum of a steady-state signal (NOM). In the case of amplitude step, the signal energy is spread over the entire observed range of frequencies. Between 25 and $75 \mathrm{~Hz}$, the spectrum is continuous, consequently it is not possible to identify a dominant fundamental tone. The definition of synchrophasor as a narrow-band component might neglect a relevant portion of signal energy. Consequently, also the performance assessment relying on TVE comparison might loose significance. For this reason, under specific transient conditions, we propose to evaluate the performance of PMUs directly in the time domain.

At each UTC-tagged time-stamp, a PMU provides an estimation of instantaneous signal amplitude, frequency and initial phase. We can use $\hat{A}, \hat{f}$ and $\hat{\varphi}_{0}$ to recover the fundamental component trend in the time domain, as follows:

$$
\hat{x}[n]=\hat{A} \cdot \cos \left(2 \pi \hat{f} n T_{s}+\hat{\varphi}_{0}\right)
$$

This procedure holds independently from the synchrophasor estimation algorithm implemented in the PMU under test. In the time domain, we can define the estimation error as the discrepancy between $\hat{x}[n]$ and the reference signal $x[n]$. We propose to quantify this discrepancy through the average quadratic index (root mean squared error) defined as follows:

$$
R M S E=\sqrt{\sum_{n} \frac{(\hat{x}[n]-x[n])^{2}}{N_{m}}}
$$




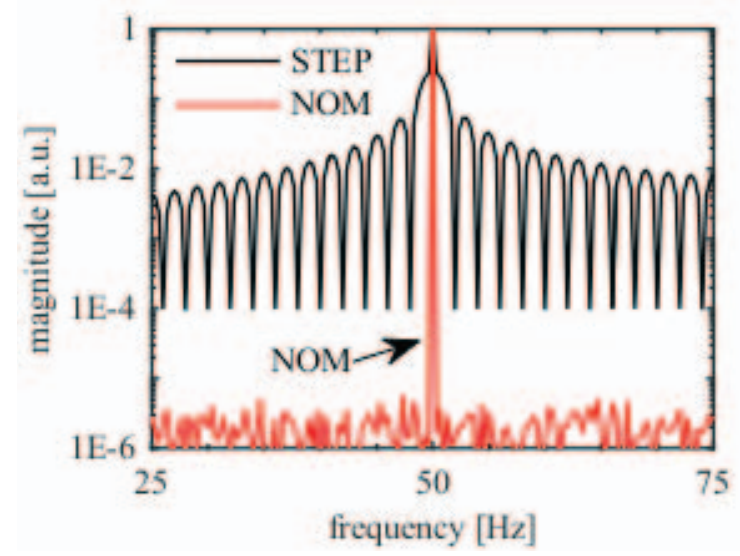

Fig. 7. Comparison between the spectrum of a amplitude step (STEP), with $k=1000$, and a steady-state signal (NOM). The step has a continuous spectrum and we cannot find a single tone in the passband $25 \div 75 \mathrm{~Hz}$.

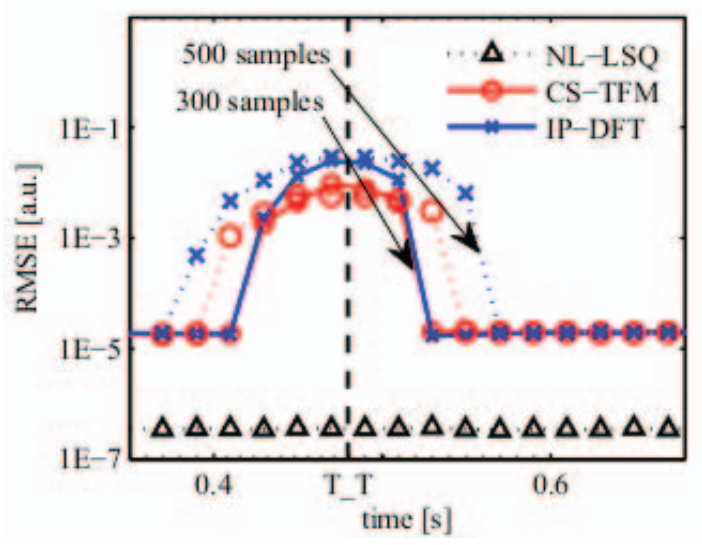

Fig. 8. RMSE comparison when an amplitude step occurs $(k=1000)$ for $60 \mathrm{~ms}$ (solid lines) and $100 \mathrm{~ms}$ (dotted lines) observation interval. In the neighbourhood of $T_{T}$, NL-LSQ shows a constant error .

We can then compare the performance of the three algorithms in terms of RMSE. We calculate the RMSE starting from the algorithm estimations. We report in Fig. 8 a RMSE comparison of the three algorithms, when an amplitude step $(k=1000)$ occurs, for both the considered observation interval lengths. As expected, NL-LSQ provides a nearly constant deviation from measurements, whereas the DFT-based algorithms significantly deviate from the optimal performances as long as the observation interval contains the transition. The RMSE index enables us also to compare generic algorithms without restricting the analysis to the fundamental component.

\section{CONCLUSION}

The first part of the paper describes the accuracy assessment of a non-linear least-squares (NL-LSQ) fitting algorithm suitable for PMU calibration. We determine the range of starting conditions which guarantees the uniqueness of the solution. We characterize the algorithm against additive wideband noise injection and study the influence of the observation interval length. Then, using synthetic waveforms, we assess the algorithm accuracy in all the static tests of the IEEE Std. In nominal conditions, assuming an uncertainty on the nominal amplitude of $4 \%$, on the fundamental frequency of $2 \mathrm{~Hz}$ and on the initial phase $\pm \pi / 4$, the fitting algorithm provides a TVE around $0.10 \mathrm{ppm}$. As expected its worst performance is obtained for OOB tests, when the interferring component is adjacent to the passband 25 to $75 \mathrm{~Hz}$, but still TVE is less than $0.30 \mathrm{ppm}$.

In the second part of the work we consider the canonical synchrophasor representation and we discuss on its validity in the presence of transient conditions. We compare the performance of NL-LSQ algorithm against two representative DFTbased algorithms, with specific reference to amplitude step test. We study the influence of step rise time on the performance of the algorithms and we propose an alternative time domain analysis. The proposed approach might be used also to compare generic algorithms, which do not restrict their analysis in the neighbourhood of the fundamental component. Future work will concern the performance assessment of NL-LSQ algorithm using signals generated by real PMU calibrators. In addition, the characterization of the algorithm will be extended to the dynamic tests of the IEEE Std and other representative transient events.

\section{ACKNOWLEDGMENT}

The research leading to the results described in this paper is part of the European Metrology Research Program (EMRP), which is jointly funded by the EMRP participating countries within EURAMET and the European Union.

\section{REFERENCES}

[1] IEEE Standard for Synchrophasor Measurements for Power Systems, IEEE Std C37.118.1-2011 (Revision of IEEE Std C37.118-2005), Dec. 2011.

[2] IEEE Standard for Synchrophasor Measurements for Power Systems Amendment 1: Modification of Selected Performance Requirements, IEEE Std C37.118.1a-2014 (Amendment to IEEE Std C37.118.1-2011), Apr. 2014.

[3] J. Braun and C. Mester, "Reference Grade Calibrator for the Testing of the Dynamic Behavior of Phasor Measurement Units," in Precision Electromagnetic Measurements (CPEM), 2012 Conference on, July 2012, pp. $410-411$.

[4] G. Stenbakken and T. Nelson, "Static Calibration and Dynamic Characterization of PMUs at NIST," in Power Engineering Society General Meeting, 2007. IEEE, June 2007, pp. 1-4.

[5] D. Colangelo, L. Zanni, M. Pignati, P. Romano, M. Paolone, J. P. Braun, and L. G. Bernier, "Architecture and Characterization of a Calibrator for PMUs Operating in Power Distribution Systems," in PowerTech, 2015 IEEE Eindhoven, June 2015, pp. 1-6.

[6] R. Garcia-Valle, G. Y. Yang, K. E. Martin, A. H. Nielsen, and J. Østergaard, "DTU PMU Laboratory Development - Testing and Validation," in 2010 IEEE PES Innovative Smart Grid Technologies Conference Europe (ISGT Europe), Oct 2010, pp. 1-6.

[7] P. Romano and M. Paolone, "Enhanced Interpolated-DFT for Synchrophasor Estimation in FPGAs: Theory, Implementation, and Validation of a PMU Prototype," IEEE Transactions on Instrumentation and Measurement, vol. 63, no. 12, pp. 2824-2836, Dec 2014.

[8] M. Bertocco, G. Frigo, C. Narduzzi, C. Muscas, and P. Pegoraro, "Compressive Sensing of a Taylor-Fourier Multifrequency Model for Synchrophasor Estimation," Instrumentation and Measurement, IEEE Transactions on, vol. 64, no. 12, pp. 3274-3283, Dec 2015.

[9] G. A. F. Seber and C. J. Wild, Estimation Methods. John Wiley \& Sons, Inc., 2005, pp. 21-89. [Online]. Available: http://dx.doi.org/10.1002/0471725315.ch2 